\title{
Covid-19 Pandemic, Glycobiology, Glycan Shields, Vaccine Strategies, Heparin Sulfate: A Mini Review
}

\author{
Steven Oppenheimer \\ Department of Biology and Center for Cancer and Developmental Biology, California State University, Northridge, United States \\ Email address: \\ steven.oppenheimer@csun.edu

\section{To cite this article:} \\ Steven Oppenheimer. Covid-19 Pandemic, Glycobiology, Glycan Shields, Vaccine Strategies, Heparin Sulfate: A Mini Review. American \\ Journal of Applied Scientific Research. Vol. 6, No. 2, 2020, pp. 46-48. doi: 10.11648/j.ajasr.20200602.14
}

Received: May 27, 2020; Accepted: June 11, 2020; Published: June 20, 2020

\begin{abstract}
The area of sugar biology (glycobiology) is an under-reported component of the Covid-19 pandemic. This minireview will provide non-experts with a brief overview of some aspects of glycobiology with emphasis on metabolic pathways and enzymes that are involved in the main topic of this review, the virus glycan shields of HIV and SARS-Cov-2 that help protect the viruses from immunological recognition. The HIV glycan shield is more dense than the SARS-Cov-2 shield and is one reason that a successful HIV vaccine has not yet been developed. The glycan shields of both HIV and SARS-Cov-2 consist of mannose chains and other sugars that resemble host molecules, explaining why they are not strongly recognized by the host's immune system as foreign. But because of the less dense SARS-Cov-2 glycan shield there is more optimism that an effective SARS-Cov-2 vaccine could be developed. This, in addition, to unusual vaccine approaches using, for example, virus messenger RNA instead of whole cells or viral proteins, and potential use of heparin sulfate to block virus attachment to cells are concepts that will be also discussed. This mini-review therefore begins with an overview of glycobiology to introduce the topic of viral glycan shields of HIV compared with SARS-COV-2. This is followed by discussion of novel vaccine approaches for SARS-COV-2 and the interesting issue of the glycan heparin sulfate that binds to the SARS-COV-2 surface and might be engineered to produce an anti-viral drug.
\end{abstract}

Keywords: Covid-19, HIV, SARS-COV-2, Glycan Shields, m-RNA Vaccines, Heparin Sulfate

\section{Introduction}

Glycobiology is a lesser reported component of viral infections such as Covid-19. Proteins and nucleic acids are well studied primarily because these fields are much simpler than sugars. While a few nucleotides or amino acids serve as building blocks for a small number of compounds, a few sugars can build many hundreds or thousands of molecules. Sugar biology is very relevant to the field of viral infections and is a focus of this mini-review.

This laboratory has studied the role of carbohydrates in 8].

embryonic development and cancer for a half century [1-

The author of this current work, Steve Oppenheimer, has been recognized by election as Fellow of the American Association for the Advancement of Science (AAAS) primarily for his work in the field of glycobiology. Studies beginning at Johns Hopkins [1] have identified metabolic pathways and specific glycans involved in cellular interactions in the developing sea urchin embryo model [27] and in cancer cells [1]. A heavily downloaded review was published by this group that covered the fundamentals of glycobiology [8]. Here, to introduce the topic of glycan shields that are manufactured by viruses, using host machinery, a brief review of aspects of glycobiology that are relevant to the issue of viral glycan shields, is first presented. Then the topic of viral glycan shields is presented with emphasis on the glycan shields of HIV and SARS-Cov-2. It will be shown that the glycan shield of HIV is very dense and is one reason why no vaccine for HIV has as yet been developed. It will be also shown that the glycan shield of the virus, SARS-Cov-2, responsible for the current Covid-19 pandemic, is less dense than that of $\mathrm{HIV}$, and therefore it may be easier to develop an effective SARS-Cov-2 vaccine [9-18]. Unusual approaches using messenger RNA vaccines are also discussed [19], as is the potential usefulness of heparin sulfate for blocking virus attachment 17-18]. 


\section{Experimental Procedure}

The experimental procedures used by papers reported in this mini-review include cell culture, inhibitor experiments, enzyme studies, cell counting methods, chromatography, mass spectrometry, model construction, cryo-EM, and glycan profiling.

\section{Results and Discussion}

This laboratory is often credited with an original seminal study that provided early evidence for a role of glycans in cellular interactions [1]. Mouse teratoma cells did not adhere in a balanced salts solution but did adhere in a more complex medium containing 51 compounds, including vitamins, cofactors, amino acids, purines, pyrimidines, nucleotides, etc. Only 1 component of the complex medium was responsible for causing the cells to adhere. That component was the amino acid L-glutamine. Through a series of experiments it was found that L-glutamine appeared to act by donating an amino group to fructose-6-phosphate forming glucosamine6-phosphate the key intermediate in the formation of glycolipids, glycoproteins and polysaccharides involved in cellular adhesiveness [1].

Many years of work, from our lab and many others, showed that glycans control adhesive interactions in many cell systems [for example from our lab 1-8]. That's the bright side of glycans. A dark side of glycans is their role in protecting some viruses from immune recognition by the host. The same molecular pathways that form the multitude of glycans that control many normal cellular functions are hijacked by some viruses to add glycans to their envelopes. These glycan coatings are termed glycan shields. Both viral glycans and normal cellular glycans are synthesized by host cells using enzymes including glycosyltransferases [9] that catalyze the addition of sugars to the growing end of sugar chains. These enzymes utilize cellular sugar nucleotides such as guanosine diphosphate mannose (GDP-mannose) and add the sugar (in this case mannose) to the growing end of a sugar chain, releasing the nucleotide (GDP) [9]. Glycosidases that remove sugars from sugar chains are also involved in glycan processing. In this way chains of mannose-type groups and other sugars are formed and these chains can comprise a glycan shield [10].

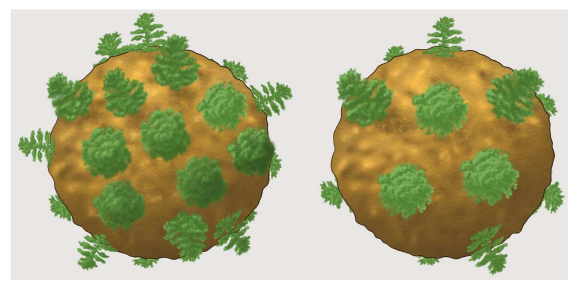

Figure 1. The glycan shields of HIV and SARS-COV-2. The green trees represent high mannose glycans on the surfaces of HIV (left) and SARSCOV-2 (right). The drawing is only intended to suggest comparative density of the glycan shields.

When a virus enters a cell, its RNA is picked up by the cell's ribosomes that produce viral proteins in the cell's endoplasmic reticulum. Cellular enzymes add sugars to the proteins at a nitrogen atom in asparagine ( $\mathrm{N}$-linked glycosylation) or at an oxygen atom in serine or threonine (O-linked glycosylation). HIV is more heavily glycosylated than SARS-Cov-2 on its spike proteins that help the viruses attach to cells (Figure 1).

This trees cartoon drawn by Rowena Oppenheimer suggests a density difference in high mannose glycans (green trees) on the HIV (left) and SARS-Cov-2 (right) surface spikes based on recent studies [10-16].

Many of the glycan shields of viruses such as HIV and SARS-Cov-2 consist of oligomannose-type chains and can also include other sugars. As these chains reach away from the virus surface and camouflage the virus, they form a barrier for early immunological recognition by the host. The more dense a glycan shield is, generally the more protected is the virus from early immunological recognition. Oligomannose-type chains and other glycans on the virus are not always exceedingly different from those in the host and therefore do not necessarily elicit a strong immunological response. That's one reason why there is still no HIV vaccine [10-16].

While glycan shields are not very antigenic, attempts are being made to produce antibodies against glycan shields, as some individuals who have been infected with HIV for years produce some antibodies against HIV glycans [11, 15]. Recent studies are precisely characterizing both HIV and SARS-Cov-2 glycans, producing important information that may help lead to better vaccine development [10-16].

Heparin sulfate is a glycan composed of sulfated sugar molecules and is present on normal cell surfaces, functioning in many cellular events such as cell adhesion and the cell cycle [17]. Heparin sulfate is present on the surfaces of most cells while heparin is only on mast cells (located in connective tissue, a type of white blood cell with immunological function). These molecules are linear polysaccharides in the glycosaminoglycan family that are covalently attached to core proteins to form proteoglycans. Different cells produce different chain lengths and different numbers of attached sulfates. This allows different heparin sulfate binding proteins to bind to different cells to regulate cellular and metabolic processes. The SARS-Cov-2 spike proteins bind to only certain heparin sulfate molecules. The best virus binding heparin sulfates had 2-4 disaccharides with 3 sulfates attached to each. The strongest binding was to compounds with 3-4 monomers. The sulfate at the 2-0 position was essential for binding, with the 6-0 sulfate also contributing to viral binding $[17,18]$. It is possible to block the anticoagulation property of heparin sulfate by selecting specific sites where sulfates can be removed. Drugs based on designer engineered heparin or heparin sulfate could be developed to help block virus attachment to cells, as these molecules competitively inhibit virus attachment [17, 18]. While glycan shields protect viruses from the host immune response, other glycans such as engineered heparin sulfate might block viral attachment to cells. 
With respect to anti-viral vaccines, in the case of SARSCov-2, an unusual approach being developed involves use of messenger RNA of the Spike protein that helps the virus attach to cells. When the viral m-RNA is in the body, the cells produce the Spike protein antigen and display it on the cell surface. The immune system produced antibody against the Spike protein. Early results suggest that this antibody provided protection and is safe and well tolerated in humans and provided full protection against viral replication in the lungs of mice. Such RNA vaccines seem to be safer than regular vaccines that use infectious elements such as whole virus particles or viral proteins. In addition, they can be much cheaper and much easier to produce in quantity than traditional vaccines [19].

This mini-review focused on the glycobiological aspects of viral infections, an under-reported area that is often overlooked, yet is of substantial importance, providing revelations about how viruses infect cells.

\section{Conclusions}

Glycobiology is an under-reported aspect of viral infections, but is important in an understanding of how viruses infect cells. Some viruses hijack the cell's machinery to produce glycan shields that can protect them from immune recognition. The glycan shield of SARS-Cov-2 is less dense than that of HIV, offering promise that a SARS-Cov-2 vaccine might be feasible. Current work on SARS-Cov-2 vaccines, including use of m-RNA, shows early promise. Heparin and heparin sulfate may be engineered to help block SARS-Cov-2 attachment to cells as these compounds can competitively inhibit virus binding to the cell surface. This mini-review is for non-experts to help in the understanding of several issues in the Covid 19 Pandemic that have not been extensively described elsewhere in such a simple compilation. Some of the approaches discussed here may offer clues that may help to solve this worldwide dilemma.

\section{Acknowledgements}

This work was supported by California State University, Northridge Department of Biology and Center for Cancer and Developmental Biology. I thank Rowena Oppenheimer for outstanding art work and Carolyn Oppenheimer for outstanding review suggestions and manuscript formatting.

\section{References}

[1] Oppenheimer, S. B., Edidin, M,, Orr, C., Roseman, S. An Lglutamine requirement for intercellular adhesion, Proceedings of the National Academy of Sciences USA, 63 (1969) pp. 1395-1402.

[2] Oppenheimer, S., Humphreys, T. Isolation of specific macromolecules required for adhesion of mouse tumor cells, Nature 232 (1971), pp. 125-127.
[3] Neri, A., Roberson, M., Connolly, D., Oppenheimer, S. Quantitative evaluation of receptor site distributions on the surfaces of specific populations of embryonic cells, Nature 258 (1975), pp. 342-344.

[4] Oppenheimer, S., Meyer, J. Carbohydrate specificity of sea urchin adhesion component, Experimental Cell Research, 139 (1982), pp. 451- 456.

[5] Idoni, B., Ghazarian, H., Metzenberg, S., Hutchins-Carroll, V, Carroll, Jr., E., Oppenheimer, S. Use of specific glycosidases to probe cellular interactions in the sea urchin embryo. Experimental Cell Research, 316 (2010), pp. 2204-2211.

[6] Aleksanyan, H., Liang, j., Metzenberg, S., Oppenheimer, S. Terminal alpha-D-mannosides are critical during sea urchin gastrulation, Zygote doi: 10.1017/SO967199416000113 (2016).

[7] Liang, J., Aleksanyan, H., Metzenberg, S., Oppenheimer, S. Involvement of L-rhamnose in sea urchin gastrulation. Part II: alpha rhamnosidase, Zygote (2016) pp. 37-377.

[8] Ghazarian, H., Idoni, B., Oppenheimer, S. B. A Glycobiology review, Acta Histochemica 113 (2011), pp. 236-247.

[9] Lairson, L. L., Henrissat, B., Davies, G. L. Withers, S. G. Glycosyl transferases: structures, functions, and mechanisms Annu Rev Biochem. 77 (2008) pp. 521-555.

[10] Watanabe, Y., Bowden, T. A., Wilson, I. A., Crispin, M. Exploitation of glycosylation in enveloped virus pathobiology, Biochim Biophys. Acta Gen Subj 1863 (2019), pp. 480-497.

[11] Crispin, M., Doores, K. J., Targeting host-derived glycans on enveloped viruses for antibody-based vaccine design Curr. Opin. Virol. 11 (2015) pp. 63-69.

[12] Seabright, G. E., Doores, K. J., Burton, Crispin, M. Protein and glycan mimicry in HIV vaccine design J. Mol Biol 431 (2019) pp. 2223-2247.

[13] Watanabe, Y., Allen, J. D., Wrapp, D., McLellan, Crispin, M. Site-specific glycan analysis of the SARS-Cov-2 spike Science 04 May (2020) DOI: 10.1126/science.abb9983.

[14] Mann, A. Spike protein for a potential vaccine https://news.uga.edu/searching-for-the covid-spike protein for a potential vaccine https:// phys.org.news/2020-04 (2020).

[15] Crispin, M., Ward, A. B., Wilson, I. A. Structure and immune recognition of the HIV glycan shield Annu. Rev. Biophys. 47 (2020), pp. 499-523.

[16] Chamary, J. V. Unlike HIV, coronavirus has a weak shield https://www.forbes.com/sites/jvchamary/2020/05/04/coronavir ud-v (2020).

[17] Thachil, J. The versatile heparin in Covid-19. Journal of Thrombosis and Haemostasis 18 (2020) https://doi.org/10.1111/jth. 14821. (2020).

[18] Liu, L., Chopra, P., Wolfert, M. A., Tompkins, S. M. SARSCov-2 spike protein binds heparin sulfate in a length and sequence-dependent manner, doi: https: www.biorxiv.org/content (2020).

[19] Moderna News Release, Moderna's work on a Covid-19 vaccine candidate (m-RNA-1273), May (2020). 